\title{
A determinação do empenhamento organizacional e do bem-estar no trabalho sobre o bem-estar subjetivo
}

\author{
The Influence of Organizational Commitment and Well-Being at Work on \\ Subjective Well-Being \\ La determinación en el compromiso organizacional y en el bienestar \\ ocupacional sobre el bienestar subjetivo
}

\author{
» Joana Vieira dos SANTOS ${ }^{1}$ (Universidade do Algarve) \\ » Gabriela GONÇALVES (Universidade do Algarve)
}

Resumo Alguns estudos apontam que, independentemente da vinculação a algum tipo de empenhamento organizacional, este construto multidimensional prevê resultados organizacionais como o desempenho, a satisfação e o bem-estar (Wright \& Davis, 2001; Wright \& Bonett, 2002). Os estudos realizados no contexto das universidades expressam que o empenhamento organizacional parece se refletir nos níveis de desempenho dos docentes universitários, não ocorrendo diferenças entre instituições públicas e privadas (Karakus \& Battal, 2009). Este estudo procurou analisar a determinação do empenhamento organizacional e do bem-estar profissional sobre o bem-estar subjetivo. A amostra foi constituída por 635 funcionários, docentes e não docentes, de instituições públicas de ensino superior portuguesas. Os resultados sugerem uma determinação positiva do empenhamento afetivo e do bem-estar no trabalho sobre o bem-estar subjetivo. Sugerem-se futuros estudos para que a temática seja aprofundada.

Palavras-chave:

Empenhamento organizacional; bem-estar subjetivo; bem-estar no trabalho.

Abstract Some studies indicate that, regardless of the attachment to some sort of organizational commitment in particular, this multidimensional construct predicts organizational outcomes such as performance, satisfaction, and well-being (Wright \& Davis, 2001; Wright \& Bonett, 2002). Studies conducted in the university setting show that organizational commitment seems to be reflected in the performance levels of academics; there was no difference between public and private universities (Karakus \& Battal, 2009). The present study sought to analyze the influence of organizational commitment and well-being at work on subjective well-being. The sample consists of 635 employees, teachers and staff of higher education public institutions in Portugal. The results suggest a positive impact of affective commitment and well-being at work on subjective well-being. We suggest future studies, in order to deepen the theme.

Keywords:

Organizational commitment; subjective well-being; well-being at work.

Resumen Algunos estudios señalan que, independientemente del vínculo con algún tipo de Compromiso Organizacional, este constructo multidimensional predice resultados organizacionales, tales como el rendimiento, la satisfacción y el bienestar (Wright \& Davis, 2001; Wright \& Bonett , 2002). Los estudios realizados en el contexto de las universidades expresan que el Compromiso Organizacional parecen reflejarse en los niveles de rendimiento de los profesores universitarios, no habiendo diferencias entre universidades públicas y privadas (Karakus \& Battal, 2009). El presente estudio buscó analizar la determinación del Compromiso Organizacional y del bienestar profesional sobre el bienestar subjetivo. La muestra está constituida por 635 empleados, profesores y administrativos, de las instituciones de enseñanza superior públicas portuguesas. Los resultados sugieren una determinación positiva del Compromiso afectivo y del bienestar en el trabajo sobre el bienestar subjetivo. Se sugieren estudios futuros, de modo a profundizar el tema.

Palabras-clave:

Compromiso organizacional; bienestar subjetivo; bienestar ocupacional

1 Endereço para contato: Departamento de Psicologia e Ciências da Educação, Faculdade de Ciências Humanas e Sociais, Universidade do Algarve, 8005-269 Faro, Portugal. Telefone: 289800 900. E-mail: jcsantos@ualg.pt 
A

s instituições de ensino superior, por missão casas de criação, promoção e divulgação do saber, assumem um papel de destaque no avanço de economias de conhecimento (Ponnuswamy \& Manohar, 2014). Na União

Europeia, a reforma do ensino superior mais notável foi iniciada pelo Processo de Bolonha, facilitando a comparabilidade entre os diversos sistemas de ensino superior e instituições de toda a Europa (Keeling, 2006). No entanto, estudos no Reino Unido, por exemplo, mostraram que as atuais políticas de ensino superior estão aumentando a mercantilização em diversas perspectivas, como a redução do financiamento direto para o ensino (Brown, 2011).

Acreditamos que as instituições de ensino superior portuguesas têm o mesmo tipo de desafio, além de enfrentar graves problemas financeiros. Considera-se que o ensino superior é uma das bases importantes para o desenvolvimento de cada país, pela premência que assume nas competências que as futuras gerações terão para lidar de modo eficaz em um meio em constante mudança. Assumindo o papel de destaque das instituições de ensino superior para a sociedade, é importante enfatizar as pessoas que nelas trabalham, bem como a forma como têm lidado com todas essas mudanças a que esses contextos têm sido sujeitos. Assim, este estudo pretende analisar, principalmente, os laços que unem esses indivíduos às organizações, bem como a sua satisfação e bem-estar.

O empenhamento parece ser importante na compreensão do comportamento dos colaboradores nas organizações (Mawday, Steers, \& Porter, 1979), em nível individual, organizacional e até mesmo na identificação dos fatores que podem contribuir para o desenvolvimento da ligação do sujeito com a organização. $\mathrm{O}$ conceito parece revestir-se de potencialidade na relação com outras variáveis (turnover, absentismo, comportamentos de cidadania organizacional) (Cunha, Rego, Cunha, \& Cabral-Cardoso, 2006). Para Caetano e Tavares (2000), a pertinência do empenhamento supera o domínio organizacional ou individual. Ou seja, os laços que unem os indivíduos às organizações têm repercussões para a própria instituição, para os sujeitos, mas também para os processos grupais envolvidos. Por isso, compreende-se por que o estudo do empenhamento desperta tanta atenção e interesse de diversos investigadores que o tornaram, e continuam a tornar, objeto de diversas investigações.

O conceito de empenhamento organizacional (commitment, na literatura anglo-saxônica) pode ter outras traduções, utilizando-se diferentes vocábulos, como comprometimento (Miguez, 1987); compromisso (Chambel, Curral, Ferreira, \& Morais, 2000) e implicação (Caetano \& Tavares, 2000). Por considerarmos que empenhamento constitui a tradução que melhor corresponde à original, e por ser utilizada por vários autores portugueses (Cabral-Cardoso, 2000; Marques, 1996; Rego, 2002, 2003), foi a opção escolhida para o presente construto.

Embora existam muitas definições de empenhamento organizacional, uma das mais reconhecidas e utilizadas é de Porter, Steers, Mowday e Boulian (1979), que o definem como a força relativa da identificação de um colaborador e do seu envolvimento em uma determinada organização. Allen e Meyer (1990, 1996) consideram-no o estado psicológico que caracteriza a ligação do indivíduo à organização, tendo implicações na sua decisão de continuar nela.

Em uma perspectiva de continuidade dos estudos de O’Reilly e Chatman (1986), Allen e Meyer (1990) desenvolveram um modelo multidimensional do empenhamento organizacional, conceitualizando-o em três componentes ou dimensões distintas, geradoras de diferentes atitudes e comportamentos: orientação afetiva, orientação instrumental e orientação normativa (Allen \& Meyer, 1996, 2000; Meyer \& Herscovitch, 2001; Meyer, Stanley, Herscovitch, \& Topolnysky, 2002). A literatura (Allen \& Meyer, 1996, 2000; Meyer \& Allen, 1997; Meyer \& Herscovitch, 2001) sugere que o empenhamento afetivo se desenvolve quando o indivíduo se sente emocionalmente conectado, identificado e envolvido na organização. Esses efeitos podem ser alcançados quando, por exemplo, o colaborador sente que a organização o trata de modo justo, deseja permanecer naquela organização, tem confiança na organização, obtém satisfação no trabalho e considera que os valores da organização têm uma orientação humanizada (Rego, Cunha, Cabral-Cardoso, \& Cunha, 2003). O empenhamento instrumental constitui o grau em que o colaborador se mantém ligado à organização devido ao seu reconhecimento dos custos associados à sua saída. Essa constatação pode vir da ausência de alternativas de emprego ou do sentimento de que os sacrifícios pessoais gerados pela saída serão elevados, ou seja, sente necessidade de permanecer naquela organização (Rego, Cunha, Oliveira, \& Marcelino, 2004). O empenhamento normativo tende a se desenvolver quando o colaborador internaliza as normas da organização por meio da socialização, dos benefícios que o induzem a atuar de modo recíproco. É possível que o sujeito 
desenvolva lealdade à organização quando se sente satisfeito e apoiado no trabalho; percebe que a organização fomenta valores próximos aos seus; confia nos líderes; e compreende que a organização adota políticas de recursos humanos que o valorizam como pessoa (Rego, 2002). Ou seja, quando o colaborador sente que deve permanecer na organização.

Os estudos de Legge (1995) sugerem que o empenhamento normativo e o empenhamento afetivo são os que contribuem de modo mais positivo para o desempenho organizacional. As pessoas com laços mais fortes nessas duas vertentes tendem a adotar mais comportamentos de ajuda aos colegas, a denotar maior iniciativa, a se preocuparem mais com a defesa da organização e a faltarem menos (Felfe \& Yan, 2009).

Os estudos realizados no contexto das universidades expressam que o empenhamento organizacional parece se refletir nos níveis de desempenho dos docentes universitários, não ocorrendo diferenças entre universidades públicas e privadas (Karakus \& Battal, 2009). No caso concreto dos professores de Hong Kong, aqueles que se encontram afetivamente empenhados revelam mais sentimentos positivos com os estudantes (love for studentes) (Choi \& Tong, 2009).

Alguns estudos apontam que, independentemente da vinculação a algum tipo de empenhamento organizacional em especial, esse construto multidimensional prevê resultados organizacionais, como o desempenho, a satisfação e o bem-estar (Wright \& Davis, 2001; Wright \& Bonett, 2002). A literatura sugere, como referido, que o sentimento de pertença e de união (e.g. Wright \& Bonett, 2002) e /ou a autonomia profissional (Ryan \& Deci, 2001) refletem-se positivamente no bem-estar.

Após alguma crise inicial na definição do bem-estar subjetivo, atualmente a perspectiva mais consensual considera que o conceito é composto por uma dimensão cognitiva, em que existe um juízo avaliativo (satisfação com a vida), e uma dimensão emocional, positiva ou negativa. A satisfação com a vida constitui uma avaliação cognitiva positiva da vida pessoal como um todo; o afeto positivo refere-se à frequência de emoções positivas em um indivíduo e o afeto negativo constitui a frequência das emoções negativas (McCullough, Heubner, \& Laughlin, 2000). Desse modo, as pessoas que demonstram bem-estar positivo experienciam uma preponderância de emoções positivas em relação às emoções negativas, e avaliam positivamente a sua vida como um todo. A satisfação geral com a vida constitui ocomponente cognitivo do bem-estar subjetivo e diz respeito ao nível de satisfação ou insatisfação com diversas dimensões e aspetos da vida pessoal (Lawler-Row \& Piferi, 2006a; Lawler-Row \& Piferi, 2006b; Zhao, Wayne, Glibkowski, \& Bravo, 2007). Os afetos positivos refletem o entusiasmo com a vida, ou seja, a tendência para experienciar emoções e sentimentos agradáveis (alegria, entusiasmo, felicidade), enquanto os afetos negativos são a medida que traduz a indisposição ou perturbação, exprimindo-se pela disposição para experimentar sentimentos e emoções desagradáveis (culpa, tristeza, ansiedade). Assim, o bem-estar subjetivo está estruturado de modo que os componentes (satisfação, afeto positivo e baixos níveis de afeto negativo) formem um fator global ou variáveis inter-relacionadas (Diener, Suh, \& Oishi, 1997; McCullough, Heubner, \& Laughlin, 2000). Por sua vez, os estudos atuais continuam contribuindo para a consistência do conceito (Diener, 2006).

Van Horn, Taris, Schaufeli e Scheurs (2004) propuseram uma estrutura do bem-estar no trabalho baseada na tradição do bem-estar psicológico, no modelo de Warr de saúde mental e nas teorias de Riff (1989) e de Riff e Keyes (1995) sobre o bem-estar geral. Os autores pressupõem que o bem-estar no trabalho consiste na avaliação positiva das várias características do trabalho e identificaram cinco dimensões para a compreensão do construto: afetiva, bem-estar profissional, bem-estar social, cansaço cognitivo e dimensão psicossomática. A dimensão Bem-Estar Profissional assume o aspecto motivacional da estrutura proposta e compreende variáveis como autonomia, aspiração e competência profissional.

O bem-estar no trabalho pode ser considerado, portanto, como a prevalência de emoções positivas no trabalho e a percepção do indivíduo de que, em suas atividades laborais, expressa e desenvolve seus potenciais e suas competências em uma aproximação às suas metas de vida. Nessa perspectiva, o bem-estar no trabalho inclui tanto aspectos afetivos (emoções e humores) quanto cognitivos (percepção de realização). Além disso, essa concepção permite que o conceito seja aplicado a diferentes contextos de trabalho, como os estudos de Warr $(1990,2008)$ indicam.

Os aspectos relativos à saúde e ao bem-estar no trabalho podem ter consequências tanto para o colaborador quanto para a organização (Danna \& Griffin, 1999). Estudos têm demonstrado benefícios do aumento do bem-estar no trabalho como na redução dos custos nos cuidados com a saúde (Whire \& Jacques, 2007); na 
diminuição do nível de estresse e de frustração (Furnham \&Walsh, 1991); na redução do absentismo (Cooper \& Dewe, 2008) e no aumento da produtividade (Helliwell \& Putnam, 2005).

Desse modo, pelo papel central que o trabalho tem na sociedade, o bem-estar profissional assume repercussões quer para o trabalho (produtividade e rendimento) (Huppert \& Whittington, 2003), quer para o sujeito (bem-estar subjetivo). Nesse sentido, é nosso objetivo compreender a determinação do empenhamento organizacional e do bem-estar profissional sobre o bem-estar subjetivo. Acreditamos que, para que as instituições de ensino superior possam ser eficientes e competitivas, elas precisam conhecer seus padrões de atuação, suas dinâmicas internas. Assim, este estudo procura constituir uma pequena contribuição para o conhecimento da realidade organizacional, de caráter mais afetivo, vivenciada no interior dessas instituições, ditas casas do saber.

\section{MÉTODO}

\section{Participantes}

A amostra foi selecionada por conveniência a partir da população que integra os ativos humanos, docentes e não docentes, de instituições públicas de ensino superior portuguesas de ambos os subsistemas (universitário e politécnico). A amostra total final foi constituída por 635 participantes de instituições de ensino superior públicas portuguesas ( $416=$ docentes; $196=$ não docentes e $23=$ não identificados). A coleta foi realizada on-line e foi utilizado como critério de inclusão a resposta a todas as questões do questionário. Os participantes foram, na sua maioria, do gênero feminino ( $\mathrm{n}=375 ; 60 \%)$, com média de idade de 39,77 $(D P=9,13)$ e as idades variaram de 23 a 64 anos. Com relação ao subsistema, 424 participantes $(66,8 \%)$ pertencem ao universitário e 211 ao politécnico $(33,2 \%)$.

\section{Instrumentos}

O questionário final apresentava uma folha de caracterização sociodemográfica, bem como os seguintes instrumentos:

a) Escala de Empenhamento Organizacional (Rego, Cunha, \& Souto, 2006). É composta por 12 itens divididos em três dimensões do empenhamento: afetivo (EA), normativo (EN) e instrumental (EI). Os itens foram criados com base na literatura (Allen \& Meyer, 1990). A escala baseia-se em uma Rating Scale com respostas alternativas específicas de 1 a $6(1=$ A afirmação não se aplica rigorosamente a mim a $6=\mathrm{A}$ afirmação aplica-se completamente a mim). Os autores (Rego et al., 2006) submeteram os dados à uma análise fatorial confirmatória. Os alfas ultrapassam o patamar de 0,70 referido por Nunnaly (1978): EA = 0,85; EN $=0,76$ e EI = 0,86. Para comparar, as médias registradas por Rego e Souto (2002) em uma pesquisa com 236 membros organizacionais portugueses foram: 4,70 (afetivo), 3,10 (normativo) e 2,80 (instrumental). Os níveis de consistência interna observados neste estudo vão ao encontro dos apresentados pelos autores da escala (afetivo: 9,854; normativo: 0,816 e instrumental: 0,844).

b) Escala de Bem-Estar no Trabalho (Gonçalves \& Santos 2010; Warr, 1990). O bem-estar afetivo no trabalho foi medido por meio do instrumento validado por Warr (1990) e corroborado para a população portuguesa por Santos e Gonçalves (2010). A escala é constituída por duas dimensões: ansiedade/contentamento e depressão/ entusiasmo, distribuídas por seis itens cada, em uma escala Rating Scale de seis níveis $(1=$ Nunca a $6=$ Sempre). Os primeiros três itens de cada dimensão estão invertidos. No que diz respeito à consistência interna, ambas as dimensões apresentam índices acima de 0,70, isto é, 0,76 para a primeira e 0,80 para a segunda.

c) Escala de Satisfação com a Vida (Satisfaction with Life Scale; SWLS). Elaborada por Diener, Emmons, Larsen e Griffins (1985), teve sua última tradução revisada e validada para a população portuguesa por Simões (1992). A Escala de Satisfação com a Vida (SWLS), um dos instrumentos mais utilizados na avaliação do componente cognitivo do bem-estar subjetivo, é composta por cinco itens, para os quais se oferece cinco níveis de resposta $(1=$ discordo muito; 5 = concordo muito). Os resultados possíveis indicam que, quanto mais elevado for o resultado obtido, maior será a satisfação com a vida. Tal como nos estudos anteriores (Diener et al., 1985; Simões, 1992), obteve-se apenas um fator, o que explica 65\% da variância. Esta escala apresenta uma consistência interna de 0,857, ainda superior à verificada pelos autores, de 0,770 (Diener et al., 1985; Simões, 1992). 
d) Escala de Atitude Afetiva da Time Attitude Scale (Galinha \& Ribeiro, 2005; Simões, 1993; Watson \& Tellegen, 1985). As escalas de afetividade positiva (PA) e afetividade negativa (NA) (Positive Affect and Negative Affect Schedule ou PANAS), desenvolvidas por Watson e Tellegen (1985), foram adaptadas para a população portuguesa por Simões (1993). Para os autores, o objetivo consistia em avaliar a dimensão afetiva do bem-estar subjetivo. A versão portuguesa, à qual nos reportamos, é composta por 22 itens. Era pedido aos indivíduos que indicassem até que ponto experienciaram os sentimentos ou os afetos indicados em cada item, em uma escala de cinco níveis $(1=$ muito pouco ou nada a $5=$ muitíssimo). Os alfas da versão original oscilaram entre 0,86 e 0,90 para a PA e entre 0,84 a 0,87 para a NA, conforme as instruções temporais.

O valor do bem-estar subjetivo é obtido a partir da subtração do valor estandardizado da afetividade positiva ao da afetividade negativa e somado com o valor padronizado da satisfação com a vida, ou seja, bem-estar = zPA - zNA + zSWLS. A aplicação dessa fórmula envolve a padronização prévia dos valores obtidos em cada uma das dimensões da escala PANAS.

\section{Procedimentos de coleta de dados e cuidados éticos}

A coleta de informações foi realizada on-line, dando ao participante maior autonomia na resposta. A clareza na apresentação do questionário on-line foi testada junto a 15 funcionários de instituições públicas de ensino superior ( 8 docentes; 7 não docentes), os quais responderam às perguntas e deram algumas sugestões de melhoria (p. ex., forma de apresentação dos itens da escala; simplificação da apresentação). Esses participantes não foram incluídos na amostra final.

Em seguida, realizaram-se os pedidos às reitorias das universidades públicas portuguesas e às presidências dos institutos politécnicos públicos portugueses, solicitando a divulgação do estudo e o respectivo pedido de colaboração junto aos funcionários das referidas instituições. Esse contato foi estabelecido através de carta registrada e por correio eletrônico. Durante três meses o questionário ficou disponível para que os funcionários das instituições pudessem acessá-lo e respondê-lo.

\section{Procedimentos de análise de dados}

Posteriormente, a informação recolhida foi organizada e analisada de acordo com os objetivos do estudo. Os dados foram processados com recurso do programa SPSS - Statistical Package for Social Sciences (v.19) e a significância foi assumida ao nível de 0,05. De modo a atender aos objetivos do estudo foram realizadas: a) médias, desvios-padrão; b) correlações de Pearson para cada uma das variáveis em estudo; c) testagem da estrutura de relação entre variáveis através de regressões hierárquicas.

\section{RESULTADOS}

O teste Kolmogorov-Smirnov revelou-se sistematicamente significativo, ou seja, a hipótese nula, que considerava que as variáveis seguiam uma distribuição normal, foi sempre rejeitada. Mais do que refletirem a não normalidade das distribuições, esses resultados devem-se ao número da amostra ( $n=635)$, já que este teste é bastante sensível a amostras de elevada dimensão. A avaliação da normalidade das distribuições foi completada pela análise dos respectivos histogramas. Salienta-se que apenas algumas variáveis revelam tendência para assimetria, ora para a direita (empenhamento afetivo; bem-estar no trabalho), ora para a esquerda (empenhamento instrumental).

Descrição dos itens: a Tabela 1 apresenta as estatísticas descritivas, média e desvio-padrão para cada dimensão. Os valores mínimo e máximo do empenhamento organizacional situam-se entre 1 e 6 , pelo que as médias observadas encontram-se sempre acima do ponto central, com maior destaque para a orientação afetiva de cada variável em estudo. Para o bem-estar no trabalho, as médias acima do ponto central indicam uma aproximação à esfera positiva, contentamento e entusiasmo, respectivamente. No que diz respeito à satisfação com a vida e à afetividade positiva, as pontuações também se apresentam acima do ponto central. 
TABELA 1. Estatísticas descritivas das variáveis em estudo $(n=635)$

\begin{tabular}{lcccc}
\hline & mínimo & máximo & $M$ & $D P$ \\
Empenhamento afetivo & 1 & 6 & 4,21 & 1,15 \\
Empenhamento normativo & 1 & 6 & 3,24 & 1,30 \\
Empenhamento instrumental & 1 & 6 & 3,17 & 1,21 \\
Bem-estar no trabalho AC & 1 & 6 & 3,49 & 1,16 \\
Bem-estar no trabalho DE & 1 & 6 & 4,56 & 1,24 \\
Satisfação com a vida & 1 & 5 & 3,32 & 0,90 \\
Afetividade positiva & 1 & 6 & 3,32 & 0,63 \\
Afetividade negativa & 1 & 5 & 1,92 & 0,65
\end{tabular}

Nota. AC - Ansiedade-Contentamento; DE - Depressão-Entusiasmo

Matriz de correlaçôes de Pearson: a matriz de correlações para cada uma das variáveis em análise pode ser observada na Tabela 2. A maioria das variáveis organizacionais consideradas está correlacionada significativamente entre si $(p=0,01)$. De forma geral, podemos afirmar que dentro de cada instrumento as diferentes dimensões tendem a se correlacionar de forma moderada, justificando, assim, sua diferenciação conceitual.

TABELA 2: Matriz de correlações de Pearson para as variáveis em estudo

\begin{tabular}{|c|c|c|c|c|c|c|c|c|}
\hline & 1. & 2. & 3. & 4. & 5. & 6. & 7. & 8. \\
\hline 1. EO Afetivo & 1 & $0,60^{* *}$ & $-0,11$ & $0,11^{\star *}$ & $0,34^{* *}$ & $0,34^{* *}$ & $0,35^{* *}$ & $-0,14^{\star *}$ \\
\hline 2. EO Normativo & & 1 & $-0,03$ & $0,02^{* *}$ & $0,24^{* *}$ & $0,25^{\star *}$ & $0,26^{* *}$ & $0,08^{*}$ \\
\hline 4. BET_AC & & & & 1 & $0,52^{* *}$ & $0,20^{* *}$ & $0,11^{* *}$ & $-0,49^{* *}$ \\
\hline 5. BET_DE & & & & & 1 & $0,41^{* *}$ & $0,32^{* *}$ & $-0,54^{* *}$ \\
\hline 8. NA & & & & & & & & 1 \\
\hline
\end{tabular}

Regressão hierárquica: a seguir, o poder de determinação das dimensões de empenhamento organizacional e de bem-estar no trabalho sobre o bem-estar subjetivo é analisado. No primeiro modelo, nota-se um efeito significativo do empenhamento sobre o bem-estar subjetivo $\left(r^{2}=21 \%\right.$; $p$ $=0,00)$. No segundo modelo, ao acrescentarmos o bem-estar no trabalho, há um aumento do poder explicativo do modelo $\left(\Delta r^{2}=19 \% ; p=0,00\right)$.

TABELA 3: Síntese da regressão hierárquica para o bem-estar subjetivo

\begin{tabular}{|c|c|c|c|c|}
\hline Modelo & Variáveis & $\beta$ & $t$ & $p$ \\
\hline & EO Afetivo & 0,31 & 7,00 & 0,00 \\
\hline \multirow[t]{3}{*}{1.} & EO Normativo & 0,07 & 1,68 & 0,09 \\
\hline & EO Instrumental & $-0,26$ & $-0,25$ & 0,00 \\
\hline & EO Afetivo & 0,18 & 4,69 & 0,00 \\
\hline \multirow[t]{4}{*}{2} & EO Normativo & 0,05 & 1,32 & 0,18 \\
\hline & EO Instrumental & $-0,11$ & $-3,53$ & 0,00 \\
\hline & BET-AC & 0,11 & 3,10 & 0,00 \\
\hline & BET-DE & 0,41 & 10,21 & 0,00 \\
\hline
\end{tabular}

Os dados sugerem especial determinação da orientação afetiva do empenhamento organizacional ( $\beta=$ $0,31 ; p=0,00)$ sobre o bem-estar subjetivo. No segundo modelo, além das duas dimensões do bem-estar no trabalho também há uma contribuição positiva e significativa. É observável ainda a contribuição da dimensão instrumental do empenhamento organizacional $(\beta=-0,11 ; p=0,00)$, no entanto, no sentido negativo.

\section{DISCUSSÃO}

No empenhamento organizacional, a dimensão com média mais elevada refere-se à afetiva. Os colaboradores que experienciam o empenhamento organizacional afetivo tendem a valorizar sua associação com a organização, 
encontrando-se mais motivados para contribuir positivamente com o desempenho organizacional (Goeddeke \& Kammeyer-Mueller, 2010; Kuvaas, 2006; Meyer \& Herscovitch, 2001; Meyer \& Smith, 2000), porque o resultado é bastante positivo.

No bem-estar no trabalho parece que os colaboradores têm preferência por um ambiente adaptativo positivo (Harris, Daniels, \& Briner, 2003; Warr, 2008). Este resultado pode ter implicações interessantes, ao nível da gestão de recursos humanos, ou seja, importa que seja garantido um ambiente tranquilo e equitativo na relação, mais do que meros momentos efémeros de alegria.

$\mathrm{Na}$ satisfação com a vida, dimensão cognitiva do bem-estar subjetivo, observam-se médias positivas, à semelhança do apontado em outros estudos (Gardiner, 2006; Vitter, Oelman, \& Wang, 2009). Na afetividade positiva e negativa, dimensão afetiva do bem-estar subjetivo, há, de igual modo, um predomínio das emoções positivas, refletindo o entusiasmo com a vida.

Em relação aos contributos explicativos para o bem-estar subjetivo experienciado, os resultados mostram que, das variáveis em estudo, as que se revestem de um caráter afetivo são as que contribuem de forma mais positiva para a predição da variável critério. Huppert et al. (2009) reconheceram que esse construto merece mais investigação, pois tem havido maior atenção ao conhecimento das condições materiais em que as pessoas vivem, em detrimento do estudo das percepões sobre a qualidade de vida (bem-estar subjetivo). De acordo com esses autores, são necessários mais estudos que retratem o bem-estar das pessoas. Por isso, desenvolveram Well-Being Module of the European Social Survey (ESS) (Huppert, et al., 2009). Importa denotar que o contexto no qual as pessoas passam tantas horas por dia deve ser fomentador de climas saudáveis e positivos, para a garantia de colaboradores também saudáveis e produtivos. É importante salientar que a tônica primordial nessas instituições em estudo são os alunos, mas para que o serviço que lhes é disponibilizado seja de qualidade, é necessário que a confiança e a identificação com a instituição se iniciem de dentro para fora, dos colaboradores para os alunos.

Este estudo procurou mostrar a determinação de variáveis organizacionais, em especial de aspecto emocional, sobre o bem-estar subjetivo dos colaboradores. O sentido de pertença não pode ser encarado como uma passagem efêmera, mas como fim em si mesmo, como um vínculo emocional efetivo, até porque o mercado escolar é people to people. Esta investigação não contemplou a perspectiva dos alunos, o que constitui uma limitação para uma melhor, e mais real, compreensão das instituições participantes. Algumas características da amostra e do próprio design transversal do estudo não permitem tirar conclusões definitivas.

Em termos futuros, seria interessante reconhecer as percepções dos clientes externos das referidas instituições, de modo a perceber se vão ao encontro das percepções dos clientes internos ou se há distinções consideráveis. Ainda, seria relevante analisar a pertença à identidade da organização, de modo a se estabelecer um contínuo relacional, desde uma perspectiva macro (identidade e cultura organizacional), até à dimensão micro (bem-estar subjetivo).

O conhecimento científico é um dado em devir, pelo que as propostas para investigações futuras seriam quase infindáveis. Desse modo, defendemos a necessidade de aprofundar esta problemática. A cultura organizacional é considerada um componente primário do funcionamento da tomada de decisão das universidades (Fralinger \& Olson, 2007), logo, pode e deve ser considerada no futuro.

\section{REFERÊNCIAS}

Allen, N. J., \& Meyer, J. P. (1990). The measurement and antecedents of affectve, continuance and normative commitment to the organization. Journal of Occupational Psychology, 63(1), 1-18. doi: 10.1111/j.2044-8325.1990.tb00506.x

Allen, N. J., \& Meyer, J. P. (1996). Affective, continuance and normative commitment to the organization: An examination of construct validity. Journal of Vocational Behavior, 49, 252-327. doi: 10.1006/jvbe.1996.0043

Allen, N. J., \& Meyer, J. P. (2000). Construct validation in organizational behavior research: The case of organizational commitment. In R. D. Goffin \& E. Helmes (Eds.), Problems and solutions in human assessement. Honoring Douglas N. Jackson at seventy (pp. 285-314). Norwell: Klumer Academic Publishers.

Andrews, F. M., \& Robinson, J. P. (1991). Measures of subjective well-being. In J. P. Robinson, P. R. Shaver, \& L. S. Wrightsman (Eds.), Atittudes, Measures of Personality and Social Psychological (Vol. 1, pp. 61-67). San Diego: Academic Press.

Brown, R. (2011). Looking back, looking forward: The changing structure of UK higher education, 1980-2012. In J. Brennan \& T. Sah (Eds.), Higher education and society in changing times: Looking back and looking forward (pp. 13-23). Center of Higher Education Research and Investigation: The Open University. 
Cabral-Cardoso, C. (2000). GRH: Evolução do conceito, perspectiva e novos desafios. In M. Cunha (Coord.), Teoria organizacional - Perspectivas e prospectivas (pp.225-249). Lisboa: Dom Quixote.

Caetano, A., \& Tavares, S. (2000). Determinantes da implicação organizacional em cinco países europeus. In M. Cabral, J. Vala, \& J. Freire (Orgs.), Atitudes Sociais dos Portugueses - Trabalho e cidadania (pp. 97-121). Lisboa: Instituto de Ciências Sociais.

Chambel, M., Curral, L., Ferreira, L., \& Morais, M. (2000). A gestão de recursos humanso e as percepções, atitudes e Bem-Estar dos trabalhadores: Um estudo comparativo de dois casos. In A. Gomes (Org.), Organizações em Transição (pp. 281-300). Coimbra: Imprensa de Universidade.

Choi, P. L., \& Tong, S. Y. (2009). Teacher commitment trends: Cases of Hong Kong teachers from 1997 to 2007. Teaching and Teacher Education, 25(5), 767-777. doi: 10.1016/j.tate.2009.01.005

Cooper, C., \& Dewe, P. (2008). Well-being, absenteeism, presenteeism, costs and challenges. Occupational Medicine, 58(8), 522544. doi: $10.1093 /$ occmed/kqn124

Cunha, M. P., Rego, A., Cunha, R. C., \& Cabral-Cardoso, C. (2006). Manual de comportamento organizacional e gestão (5a ed.). Lisboa: RH Editora.

Danna, K., \& Griffin, R. W. (1999). Healthy and well-being in the workplace: a review and synthesis of the literature. Journal of Management, 25(3) , 357-384. doi: 10.1177/014920639902500305

Diener, E. (2006). Guidelines for national indicators of subjective well-being and ill-being. Journal of Hapiness Studies, 7(4) , 397404. doi: 10.1007/s10902-006-9000-y

Diener, E., Emmons, R. A., Larsen, R. J., \& Griffin, S. (1985). The satisfaction with life scale. Journal of Personality Assessment, 49(1), 71-75. doi: 10.1207/s15327752jpa4901_13

Diener, E., Suh, E., \& Oishi, S. (1997). Recent findings on subjective well-being. Indian Journal of Clinical Psychology , 24(1), 2541.

Felfe, J., \& Yan, W. H. (2009). The impact of workgroup commitment on organizational citizenship behaviour, absenteeism and turnover intention: The case of Germany and China. Asia Pacific Business Review, 15(3) , 433-450. doi: $10.1080 / 13602380802667411$

Fralinger, B., \& Olson, V. (2007). Organizational culture at the university Level: A study using the OCAI instrument. Journal of College Teaching \& Learning, 4(11), 85-98.

Furnham, A., \& Walsh, J. (1991). Consequences of person environment incongruence: Absenteeism, frustration and stress. Journal of Social Psychology, 131, 187-204.

Galinha, I. C., \& Ribeiro, J. L. P. (2005). Contribuição para o estudo da versão portuguesa da Positive and Negative Affect Schedule (PANAS): Il estudo psicométrico. Análise Psicológica, 2(23), 219-227.

Gardiner, K. (2006). The effects of personalit and perceived stress on the well-being of nursing and psychology students: A cross sectorial study (Dissertação de mestrado não publicada). Universidade de Edimburgo, Escócia.

Goeddeke, J. F., \& Kammeyer-Mueller, J. (2010). Perceived Support in a dual organizational environment: Union participation in a university setting. Journal of Organizational Behavior, 31(1), 65-83. doi: 10.1002/job.629

Harris, C., Daniels, K., \& Briner, R. B. (2003). A daily diary study of goals and affective well-being at work. Journal of Occupational and Organizational Psychology, 76(3), 401-410. doi: 10.1348/096317903769647256

Helliwell, J., \& Putnam, R. D. (2005). The social context of well-being. In F. A. Huppert, B. Keverne, \& N. Baylis (Eds.), The science of well-being (pp. 435-459). Oxford: Oxford University Press.

Huppert, F. A., \& Whittington, J. E. (2003). Evidence for the independence of positive and negative well-being: Implications for quality of life assessment. British Journal of Health Psychology, 8(1), 107-122.

Huppert, F. A., Marks, N., Clark, A., Siegrist, J., Stutzer, A., \& Vitterso, J. (2009). Measuring well-being across Europe. Social Indicators Research, 91, 301-315. doi: 10.1007/s11205-008-9346-0

Karakus, M., \& Battal, A. (2009). Teachers' commitment focuses: A three-dimensioned view. Journal of Management Development, 28(5), 425-438. doi: 10.1108/02621710910955967

Keeling, R. (2006). The Bologna process and the Lisbon research agenda: The european commission's expanding role in higher education discourse. European Journal of Education, 41(2), 203-223.

Kuvaas, B. (2006). Work performance, affective commitment, and work motivation: The roles of pay administration and pay level. Journal of Organizational Behavior, 27(3), 365-385. doi: 10.1002/job.377

Lawler-Row, K. A., \& Piferi, R. L. (2006a). Life well lived? Personality and Individual Differences, 41(6), 1009-1021. 
Lawler-Row, K. A., \& Piferi, R. L. (2006b). The forgiving personality: Describing a life well lived? Personality and Individual Differences, 41(6), 1009-1020. doi: 10.1016/j.paid.2006.04.007

Legge, K. (1995). Human resource management: Rhetorics and realities. London: Macmillan Business.

Marques, C. (1996). Comportamento Organizacioanl e Gestão: Perspectivas e aplicação. In C. Marques \& M. Cunha (Orgs.), Comportamento Organizacional e Gestão de Empresas (pp. 13-41). Lisboa: Dom Quixote.

Mawday, R., Steers, R., \& Porter, L. (1979). The measurement of organizational commitment. Journal of Vocational Behavior, 14(2), 224-247.

McCullough, G., Heubner, E. S., \& Laughlin, J. (2000). Life events, self-concept and adolescents' positive subjective well-being. Psychology in Schools, 37, 281-291. doi: 10.1002/(SICI)1520-6807(200005)37:3<281::AID-PITS8>3.0.CO;2-2

Meyer, J. P., \& Allen, N. J. (1997). Commitment in the workplace: Theory, research and application. Newbury Park, CA: Sage.

Meyer, J. P., \& Herscovitch, L. (2001). Commitment in the workplace: Toward a general model. Human Resource Management Review, 11(3), 299-326. doi: 10.1016/S1053-4822(00)00053-X

Meyer, J. P., \& Smith, A. C. (2000). HRM practices and organizational commitment: Test of a mediation model. Canadian Journal of Administrative Sciences, 17(4) , 319-331. doi: 10.1111/j.1936-4490.2000.tb00231.x

Meyer, J. P., Stanley, D. J., Herscovitch, L., \& Topolnytsky, L. (2002), Affective, continuance and normative commitment to the organization: A meta-analysis of antecedents, correlates, and consequences, Journal of Vocational Behavior, 61(1), 20-52. doi: 10.1006/jvbe.2001.1842

Miguez, J. (1987). Satisfação no trabalho e comportamentos de ausência. Revista de Psicologia e Ciências da Educação, 2, 1735.

O’Reilley, C., \& Chatman, J. (1986). Organizational commitment and psychological attachment: The effects of compliance, identification and internalization on prosocial behaviour. Journal of Applied Psychology, 71(3), 429-499.

Ponnuswamy, I., \& Manohar, H. L. (2014). Impact of learning organizational culture on performance in higher education institutions. Studies in Higher Education, 1-14. doi: 10.1080/03075079.2014.914920

Porter, L., Steers, R., Mowday, R., \& Boulian, P. (1979). Organizational commitment, job satisfaction and turnover among psychiatric technicians. Journal of Applied Psychology, 51, 603-609.

Rego, A. (2002). Comportamentos de cidadania dos professores universitários: Um constructo com promissora família. Psicologia: Organizações e Trabalho, 2(1), 63-69.

Rego, A. (2003). Empenhamento Organizacional e ausência psicológica. Revista de Administração de Empresas, 43(4), 25-35.

Rego, A. \& Souto, S. (2002). Comprometimento organizacional: Um estudo luso-brasileiro sobre a importância da justiça. Anais do XXVI Encontro da ANPAD (Associação Nacional dos Programas de Pós-Graduação em Administração), Salvador, Brasil, 22-25 Setembro. (CDROM)

Rego, A., Cunha, R. C. e, Cabral-Cardoso, C., \& Cunha, M. e P. (2003). Comportamento organizacional e gestão. Lisboa: RH Editora.

Rego, A., Cunha, M., \& Souto, S. (2006). Espiritualidade nas organizações e Empenhamento Organizacional: Um estudo empírico (Documentos de Trabalho em Gestão DEGEI, G/1/n6, pp. 1-30). Recuperado de: https://www.ua.pt/degei/readobject. aspx?obj=7982

Rego, A., Cunha, M. P. e, Oliveira, C. M., \& Marcelino, A. R. (2004). Coaching para executivos. Lisboa: Escolar Editora.

Riff, C. D. (1989). Happiness ir everything, or is it? Explorations on the meaning of psychological well-being. Journal of Personality and Social Psychology, 57(6), 1069-1081.

Riff, C. D., \& Keyes, C. L. (1995). The structure of psychological well-being revisited. Journal of Personality and Social Psychology, 69(4), 719-729.

Ryan, R. M., \& Deci, E. L. (2001). To be happy or to be self-fulfilled: A review of research on hedonic and eudaimonic well-being. In S. Fiske (Ed.), Annual Review of Psychology (Vol. 52, pp. 141-166). Palo Alto, CA: Annual Reviews/ Inc.

Santos, J., \& Gonçalves, G. (2010). Contribuição para a adaptação portuguesa da escala de Bem-Estar no Trabalho de Warr (1990). Psicologia, Educação e Cultura, 24(2), 285-300.

Simões, A. (1992). Ulterior validação de uma escala de satisfação com a vida (SWLS). Revista Portuguesa de Pedagogia, 26(3), 503-515.

Simões, A. (1993). São os homens mais agressivos do que as mulheres? Revista Portuguesa de Pegagogia, 27(3), $387-404$. 
Van Horn, J. E., Taris, T. W., Schaufeli, W. B., \& Scheurs, P. J. (2004). The structure of occupational well-being: A study among Dutch teachers. Journal of Occupational and Organizational Psychology, 77(3), 365-375 doi: 10.1348/0963179041752718.

Vitter, J. C., Oelman, H. I., \& Wang, A. L. (2009). Life satisfaction is not a balanced estimator of the good life: Evidence from reaction time measures and self-reported emotions. Journal of Hapiness Studies, 10(1), 1-17. doi: 10.1007/s10902-0079058-1

Warr, P. B. (1990). The measurement of well-being and other aspects of mental health. Journal of Occupational Psychology, 63(3), 193-210. doi: 10.1111/j.2044-8325.1990.tb00521.x

Warr, P. B. (2008). Environmental vitamins, personal judgments, work values and happiness. In S. Cartwright \& C. L. Cooper (Eds.), The Oxford Hanbook of Organizational Well-being (pp. 57-85). Oxford: Oxford University Press.

Watson, D., \& Tellegen, A. (1985). Toward a consensual structure of mood. Psychological Bulletin, 98(2), 219-235.

Whire, K., \& Jacques, P. (2007). Combined diet and exercise intervention in the workplace. American Association of Occupational Health Nurses, 55(3), 109-114.

Wright, B. E., \& Davis, B. S. (2001). Job satisfaction in public sector: The role of work environment. American Review of Public Administration, 33(1), 70-90. doi: 10.1177/0275074002250254

Wright, T., \& Bonett, D. (2002). The moderating effects of employee tenure on the relations between organizational commitment and job performance: A meta analysis. Journal of Applied Psychology, 87(6), 1183-1190. doi: 0.1037/0021-9010.87.6.1183

Zhao, H., Wayne, S. J., Glibkowski, B. C., \& Bravo, J. (2007). The impact of psychological contract breach on work-related outcomes: A meta-analysis. . Personnel Psychology, 60, 647-680. doi: 10.1111/j.1744-6570.2007.00087.x 